\title{
CORRESPONDENCE
}

\section{Challenges for Family Medicine and for Family Physicians}

To the Editor: One of the challenges for family medicine and for family physicians who are committed to research and publishing is that our discipline, more than almost any other, is practice-based. Unlike many medical and surgical subspecialties, we were not born in a laboratory. Following this line of logic, it is clear that much of the research that attempts to answer important questions in family medicine must be done in our community-based practices. This reality creates a dilemma, because most trained and experienced researchers and writers in family medicine are based in medical schools or residencies, not in the real-world practice sites that could help answer our questions.

Several approaches have been successfully used to deal with the frequently encountered mismatch in location between practice-oriented family physicians and research-oriented family physicians. One of the best known approaches has been the regional (or national) research network, in which a large number of practices have been linked to an academic center (or network hub), to address problems in an organized fashion. The recent Edwards and Norris article ${ }^{1}$ illustrates another solution to the same problem. In this case, an inquisitive rural family physician in full-time practice contacted his regional medical school (Idaho is a participant in the University of Washington's affiliated family practice residency network) seeking a collaborator. Once the right "match" was made, the community practice was able to provide the data, and the medical school faculty member was able to assist with research design, data analysis, and writing. This sort of partnership has great potential in linking the community practice-based and the academic centerbased members of our specialty. It offers a powerful model to assist in answering important practical "realworld" questions.

Thomas E. Norris, MD Department of Family Medicine University of Washington Physicians Network University of Washington Seattle

\section{References}

1. Edwards JK, Norris TE. Colonoscopy in rural communities: can family physicians perform the procedure with safe and efficacious results? J Am Board Fam Pract 2004;17: $353-8$.

\section{The Impact of Clinical Practice Guidelines Should Not Be Overestimated}

To the Editor: Compliance with published clinical practice guidelines (CPG) has been reported to be a problem worldwide. A recent study by $W$ olfe et $\mathrm{al}^{1}$ concluded that most American family physicians find CPGs to be help- ful, and familiarity with CPGs is fairly uniform across most subgroups studied.

We recently performed a study in Estonia on doctors compliance with a CPG for type 2 diabetes mellitus (DM). ${ }^{2,3}$ We studied 354 family doctors, a random sample from the list of all family doctors in Estonia: 163 responded (response rate 46\%). Respondents reported the guidelines were available in $76 \%$ of cases, which is about 3 times higher than reported by Wolfe et al. ${ }^{1}$ Nevertheless, our results on how doctors change their practices when CPGs are available at their offices are not as encouraging those reported by Wolfe et al. ${ }^{1}$ In the that study, it was found that $28 \%$ to $33 \%$ of the respondents reported they had changed their practice as a result of CPGs. The study also showed that of the subgroup of doctors who were aware of CPGs, $81 \%$ to $91 \%$ changed patient care. In our study, performed 3 years after the guideline was approved and distributed, we found that there was a great variability in doctors' self-reported performance, depending on the guideline recommendation, and the results are far from what we expected (Table 1).

We found no relationship between the use of a CPG for DM and the location of practice (rural or urban) or whether the practice was solo or group. In our study, the more experienced doctors reported better availability and better use of the DM CPG compared with their younger colleagues, which is the opposite of the results in the study of American doctors.

It has been reported in many studies that the attitudes of family physicians toward CPGs depends on varied circumstances, including cultural and legal factors. Lack of compliance with the guidelines may indicate deficiencies in the physician's knowledge, implementation problems, lack of belief in the guidelines, or problems in patient compliance. ${ }^{4}$ Another possible explanation is that doctors involved in surveys might have overestimated changes in their practice. It would be useful to study the patients to detect the actual change in practice.

Margus Lember, MD

Anneli Rätsep, MD

Department of Polyclinic and Family Medicine

University of Tartu Tartu, Estonia

\section{References}

1. Wolfe RM, Sharp LK, Wang RM. Family physicians' opinions and attitudes to three clinical practice guidelines. J Am Board Fam Pract 2004;17:150-7.

2. A desktop guide to Type 2 diabetes mellitus. European Diabetes Policy Group 1999. Diabet Med 1999;16:716-30.

3. Estonian Society of Family Doctors. CPG on type 2 diabetes (2. tüüpi suhurtõve ravijuhised). Estonian Society of Family Doctors; 2000. 
4. Lawler FM, Viviani N. Patient and physician perspectives regarding treatment of diabetes: compliance with practice guidelines. J Fam Pract 1997;44:369-73.

\section{Author's Reply}

\section{Compliance with Clinical Guidelines and the 'Law of Thirds'}

To the Editor: We read with interest the comments of Drs. Lember and Rätsep on their study of diabetes mellitus clinical practice guidelines in Estonia. Although their results contradicted our findings in the United States, we suggest that the differences are largely accounted for by methodological differences in the study design.

Diabetes mellitus $(\mathrm{DM})$ is a common disorder that affects all ages and is associated with significant morbidity if not properly treated. In addition, it is highly likely that most family doctors have daily or weekly exposure to patients with DM. Access and motivation to read DM guidelines may have been affected by these facts. In our study, the focus was upon 4 age-specific guidelines, with the realization that family doctors would vary in the number of patients they treated with each clinical problem. Motivation to read the guideline might be affected by the number of patients that are seen with that clinical condition as well as the potential impact of mistreating the condition.

Similarly, our study asked the doctor to respond to a very general question, "Have you changed your practice as a result of reading the guideline?" Although more respondents in our study stated that they had changed practice, the social desirability and general tone of the question makes it easy to respond affirmatively.

It is our opinion that the differences in study results highlight the limitations of both study designs-reliance upon self-report as opposed to observation of actual practice. As is the case in many areas of science, financial restrictions adversely affect research designs.

The low rates of overall compliance in both of these articles are not surprising. A number of studies have shown poor correlation between either continuing medical education (CME) or clinical practice guideline (CPG) dissemination and actual changes in patterns of care in clinical settings. ${ }^{1,2}$ Our study found that approximately one third of the family physicians we studied were very aware of the guidelines, one third were somewhat aware of the guidelines, and one third were unaware of them. ${ }^{3}$ We would extend this to a general rule, probably applying to most specialties- the "law of thirds." In medicine, as in most areas of life, there is a range of knowledge among the practitioners. The family physicians who are in the upper third probably have the most interest and/or ability in reading, learning, and keeping current, and probably the most flexibility in adapting their practice patterns to new changes. They will learn aggressively whether or not there are $\mathrm{CME}$ requirements or new CPGs showing up in their mailbox. The middle third will maintain some level of currency, but are probably more focused on clinical practice. Their currency will remain moderate regardless of CME or CPG requirements. Finally, the lowest third will do their best to keep up while maintaining a busy practice. They will meet their $\mathrm{CME}$ requirements, but this will not produce significant changes in their practice.

What is a guideline? It is essentially a well-researched and authoritative review article that has the blessing of the body promoting it. As such, it is meant not only to be a good review article, but also to set a standard of care. Hopefully, it is has more practical point-of-care usefulness in its organization and presentation than a good review article, but this is not always the case. As with any review article, how well physicians use it will ultimately reflect their pre-existing scholastic qualities-i.e. which "third" they reside in. Indeed, in our study, the rates of self-reported change in practice correlated closely with which "third" reported the change; we suspect that had Lember and Rätsep segregated their group by level of familiarity with the diabetes guideline, as opposed to availability, a similar trend would have been found.

There has been a great deal of research about how to improve physicians' compliance with CPGs, but we believe that gentle reminders at the point of care, possibly linked to financial performance incentives, are the most likely to work. ${ }^{4}$ It may be that a gradual shift to electronic medical records will make this more practical in the future.

Robert M. Wolfe, MD

Lisa K. Sharp, $\mathrm{PhD}$

Department of Family Medicine The Feinberg School of Medicine

Northwestern University

Chicago, IL

\section{References}

1. Davis DA, Thomson MA, Oxman AD, Haynes RB. Changing physician performance: a systematic review of the effect of continuing medical education strategies. JAMA 1995;274: 700-5.

2. Davis DA, Taylor-Vaisey AL. Translating guidelines into practice. A systematic review of theoretic concepts, practical experience and research evidence in the adoption of clinical practice guidelines. CMAJ 1997;157:408-16.

3. Wolfe RM, Sharp LK, Wang RM. Family physicians' opinions and attitudes to three clinical practice guidelines. J Am Board Fam Pract 2004;17:150-7.

4. Phillips LS, Branch WT, Cook CB, et al. Clinical inertia. Ann Intern Med 2001;135:825-34. 